\title{
Numerical Analysis and Optimization on Vortex Shedder by the Linearity of Strouhal Number against Reynold Number
}

\author{
Su Myat Nyein and He Xu
}

\begin{abstract}
The design parameters and blockage ratio of the bluff body called vortex shedder of the vortex flowmeter strongly influence on the flowmeter performance. The released frequency only depends on the size of vortex shedder and flow rate of fluid while independent of the fluid properties. In the condition of constant Strouhal number, the flowmeter is not affected by the properties such as temperature or pressure of measuring fluids. Thus, optimization a flowmeter design by considering the linearity of Strouhal number against the Reynold number becomes one of the ideas of designing the flowmeter. In the present work, the unsteady flow past a bluff body of diamond-shape with slit has been carried out to analysis on the Strouhal number using FLUENT, a CFD code. The numerical analysis was conducted for non-circular cross-section pipe of $7.457 \mathrm{~mm}$ hydraulic diameter in the Reynold number range of 2400 to 60000 . The blockage ratio from 0.16 (about 1/6) to 0.30 (about $1 / 3$ ) were considered and for the slit ratio, $0.10-0.16$ were used. We took only three angle, 450,600 , and 90o for this work as longer and more slender shedding bodies result in weaker and less coherent shedding. The optimized design was achieved by the linearity of Strouhal number against Reynold number. And, the linearity is determined by the error percentage of Strouhal number. According to the results, it has been found that the bluff body with the parameters of 0.24 blockage ratio, 0.14 slit ratio and 600 apex angle is the best design.
\end{abstract}

Index Terms - Linearity of Strouhal number against Reynold number, diamond-shape with slit, non-circular cross-section pipe, numerical analysis.

\section{INTRODUCTION}

Vortex flowmeter is one kind of flowmeter based on Karman vortex street theory. It uses a sensor to detect the frequency of the fluid caused by flowing through the vortex shedder in the pipe and then measures the flow of fluid. The frequency depends on the size of vortex shedder and flow rate of fluid, while independent of the fluid parameters, such as the temperature, pressure. It can be expressed by the following formula:

Manuscript received March 23, 2015; revised July 23, 2015. This work was supported by the National Natural Science Foundation of China under Grant 60775060, Specialized Research Fund for the Doctoral Program of Higher Education under Grant 200802171053, 20102304110006, 20122304110014, Natural Science Foundation of the Heilongiiang Province of China under Grant F200801 and Harbin Science and Technology Innovation Talents Special Fund under Grant 2012RFXXG059.

The authors are with the College of Mechanical and Electrical Engineering, Harbin Engineering University, Harbin, 150001, P.R. China (e-mail: sumyatnyein.me@gmail.com, railway_dragon@sohu.com).

$$
f=S t U_{i} / d
$$

where, $f$ is the frequency of Karman Vortex Street, $S t$ is Strouhal number, $U_{i}$ is fluid velocity, and $d$ is width of vortex shedder.

The bluff body called vortex shedder is a primary element of the vortex flowmeter. The geometrical design parameters and blockage ratio of the vortex shedder strongly affect on the vortex flowmeter performance. In the first design, circular cylinder was used as the vortex shedder. However, it was found that the circular cylinder cannot give the fixed separation point in the range of Reynold number [1]. The linearity of Strouhal number requires the location of the separation point to be fixed regardless of Reynolds number. With this reason, most modern flowmeter have used the non-circular cross-section bluff bodies and sharp-edges bluff bodies in order to remove the shifts resulting from changes in the boundary layer. Attempts of many researchers to design the bluff body with sharp edges can be seen in the primary element section of reviews by [2], [3] .

Although the vortex shedding flowmeters are widely used in many fields of industry, it can be seen that neither ideal design of flowmeter nor universal bluff body was found. The idea of designing a flowmeter based on Strouhal number against Reynolds number as constant was first suggested by [4]. The constant Strouhal number means that the flowmeter is not affected by the properties such as temperature or pressure of measuring fluids. Ref. [5] carried out an experiment with trapezoidal-shape bluff body to optimize a flowmeter design by considering the linearity of Strouhal number against the Reynold number. The experimental study by [6] with three different shape (trapezoidal cylinder, circular with slit and triangular-semicylinder consisting of a slit between these two parts) highlighted that the two shedder with a slit can significantly reduced the pressure loss. Moreover, these two shedders are superior in linearity to the trapezoidal cylinder. [7] investigated numerically on the effect of bluff body shape on vortex flowmeter performance. The vortex flowmeter requires a body shape with sharp corners to generate stable vortex shedding frequency. Thus, they selected a triangular shape of $60^{\circ}$ angle with splitter plate or a diamond shape as optimum designs that generate stable vortex shedding frequency with low coefficients of permanent pressure loss and drag. However, the Strouhal number did not evaluated.

In this paper, the unsteady flow past a bluff body of diamond shape with slit have been analyzed using FLUENT, a computational fluid dynamics (CFD) code. The works 
[5]-[7] force us to choose the diamond shape with slit (or two triangular shapes their base edges are facing with each other) as the interest body shape for this simulation. The numerical analysis was conducted for non-circular pipe of $7.457 \mathrm{~mm}$ hydraulic diameter in the Reynold number range of 2400 to 60000. The optimized design was achieved by the linearity of Strouhal number against Reynold number. And, the linearity is determined by the error percentage.

\section{Numerical ANALYSIS METHODOlOGIES}

FLUENT is a computational dynamic code capable of modeling fluid flow and heat transfer. The supporting numerical methods adopt the governing equations for conservation in integral form and use the control-volume-based technique. This technique consists of division of the domain into discrete control volumes using a computational grid and integration the governing equations on each control volumes [8].

\section{A. Mean Flow Equations}

The continuity equation and unsteady momentum equation are:

$$
\begin{gathered}
\rho \frac{\partial}{\partial x_{i}}\left(U_{i}\right)=0 \\
\rho \frac{\partial}{\partial t}\left(U_{i}\right)+\rho \frac{\partial}{\partial x_{j}}\left(U_{i} U_{j}\right)=-\frac{\partial(p)}{\partial x_{i}}+\frac{\partial}{\partial x_{j}}\left(\mu_{e f f} \frac{\partial\left(U_{i}\right)}{\partial x_{j}}\right)
\end{gathered}
$$

\section{B. Turbulent Model Used}

The standard $k-\varepsilon$ turbulence model is used in this study. The unsteady transport equations for $k$ and $\varepsilon$ can be written as:

$$
\begin{gathered}
\rho \frac{\partial(k)}{\partial t}+\rho \frac{\partial\left(U_{i} k\right)}{\partial x_{i}}=\frac{\partial}{\partial x_{i}}\left[\left(\mu+\frac{\mu_{t}}{\sigma_{k}}\right) \frac{\partial(k)}{\partial x_{i}}\right]+P_{k}-\rho(\varepsilon) \\
\rho \frac{\partial(\varepsilon)}{\partial t}+\rho \frac{\partial\left(U_{i} \varepsilon\right)}{\partial x_{i}}= \\
\frac{\partial}{\partial x_{i}}\left[\left(\mu+\frac{\mu_{t}}{\sigma_{\varepsilon}}\right) \frac{\partial(\varepsilon)}{\partial x_{i}}\right]+\frac{k}{\varepsilon}\left(C_{1 \varepsilon} P_{k}-C_{2 \varepsilon} \rho(\varepsilon)\right)
\end{gathered}
$$

where $k$ is turbulent kinetic energy $(\mathrm{Nm}), \mu_{t}$ is eddy/turbulent viscosity (Nsm-2), $\sigma_{k}$ is turbulent Prandtl number for $k, \sigma_{\varepsilon}$ is turbulent Prandtl number for $\varepsilon, P_{k}$ is generation term , $\varepsilon$ is turbulent dissipation rate $(\mathrm{Nm} 2 \mathrm{~kg}-1)$, $C_{1 \varepsilon}$ and $C_{2 \varepsilon}$ are turbulence model constants.

The generation term $P_{k}$ has the following form:

$$
P_{k}=\mu_{t} \frac{\partial\left(U_{i}\right)}{\partial x_{j}}\left(\frac{\partial\left(U_{i}\right)}{\partial x_{j}}+\frac{\partial\left(U_{j}\right)}{\partial x_{i}}\right)
$$

The turbulent viscosity is calculated as:

$$
\mu_{t}=\rho c \mu \frac{(k)^{2}}{(\varepsilon)}
$$

The model constant are use, by default, as $C_{\mu}=0.09, C_{1 \varepsilon}=1.44, C_{2 \varepsilon}=1.92, \sigma_{k}=1.0$, $\sigma_{\varepsilon}=1.3$ and the effective viscosity $\mu_{e f f}$ is obtained by:

$$
\mu_{\text {eff }}=\mu+\mu_{t}
$$

\section{Geometry of Analysis}

Fig. 1 shows the geometry of interested body taken for this simulation. A bluff body with apex angle $(\theta)$, width $(d)$ and slit $(s)$ is placed at a distant 2D from the inlet and the outlet is sufficient far downstream from the bluff body in order to avoid the unnecessary effects of inlet and exit, where $D$ is the pipe diameter.

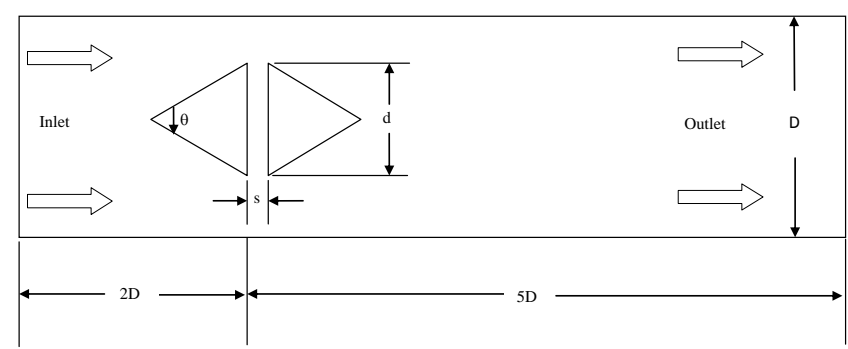

Fig. 1. Flow domain and Interested Bluff Body Shape (not to scale).

\section{Meshing}

The preprocessor software package GAMBIT is employed in meshing. The total number of grid cells is about 150000 and was obtained by using Quad/Tri - Pave meshing scheme.

\section{E. Boundary Conditions}

In the present case, velocity at the inlet and outflow were specified as the boundary conditions. The velocity inlet profile was set to a constant value and the hydraulic diameter $\left(D_{h}\right)$ and turbulent intensity $(I)$ were used as turbulent quantities. The turbulent intensity $(I)$ is calculated from the following formula.

$$
I=0.16\left(\operatorname{Re}_{D_{h}}\right)^{-1 / 8}
$$

No-slip boundary conditions are applied to the surface of bluff body as well as to the top and the bottom of the domain.

\section{F. Validation of the Code}

As the shedder design used in this simulation is new, there are no data in the literature about the flow analysis with exactly same bluff body design. Johnsson et al. [9] have done the numerical simulation using a $k-\varepsilon$ turbulence model. They used the triangular cylinder with 0.33 blockage ratio as bluff body and found that the flow behind triangular-shaped body is unsteady with Strouhal number of 0.27 numerically 
(an experimental value of 0.25 ). In the present work, the Strouhal number of dual triangular-shaped body with blockage 0.3 at $\mathrm{Re}=50000$ is 0.28 which is reasonable agreement with the Johnsson's data.

\section{Simulation Results}

Numerical investigation on unsteady flow past a bluff body of diamond shape with slit has been carried out to analysis on the Strouhal number. To accurately calculate the shedding frequency, an average value of 10 shedding cycles was taken. The time derivative scheme is the same in Johnsson's paper [9]. The first order fully implicit and sufficient small time steps (90 time steps per one shedding cycle) are used in order to minimize the discretization error.

This study was made by changing the ratio of vortex shedder diameter $(d)$ to pipe diameter $(D)$, the ratio of slit $(s)$ to shedder diameter $(d)$ and the apex angel $(\theta)$. The linearity of the Strouhal number is determined by the error percentage and the error $(e)$ has a function of

$$
e=\frac{1}{N} \sum_{i=1}^{N} \frac{\left|S t_{i}-\overline{S t}\right|}{\overline{S t}}
$$

\section{A. Analysis on Blockage Ratio (d/D Ratio)}

At first, the blockage ratio was analyzed while the other parameters such as slit and apex angle are kept constant. The bluff bodies with blockage ratio of from 0.16 to 0.30 were considered in the present work. The Linearity of Strouhal number (St) against Reynold number (Re) with different blockage ratios is shown in Fig. 2. Here, the Reynold number is based on hydraulic diameter of pipe. It was found that 0.25 is the most linearity among these considered ratios. Thus, this blockage ratio 0.25 was selected as optimum in general. Also it is reasonable with the reported blockage ratios [10].

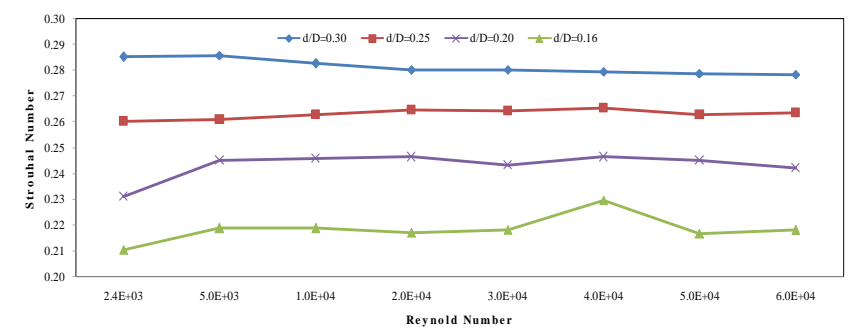

Fig. 2. Linearity of St against Re with different blockage ratio $(d / D=0.16 \sim$ $d / D=0.30)$

TABLE I: MEAN STROUHAL NUMBER AND ERROR (\%) FOR MORE PRÉCISED VALUES

\begin{tabular}{|c|c|c|}
\hline $\begin{array}{r}\text { Blockage ratio } \\
d / D\end{array}$ & $\begin{array}{l}\text { Mean Strouhal } \\
\text { number } \\
\overline{S t}\end{array}$ & $\begin{array}{l}\text { Error }(\%) \\
e\end{array}$ \\
\hline 0.23 & 0.2592 & $0.458^{*}$ \\
\hline 0.24 & 0.2598 & $0.404 *$ \\
\hline 0.25 & 0.2631 & 0.518 \\
\hline 0.26 & 0.2640 & $0.474^{*}$ \\
\hline 0.27 & 0.2674 & 1.075 \\
\hline
\end{tabular}

* The selected ratios for further studying on slit effect.

To get the more précised ratio, we tried to observe on four ratio proximity to 0.25 which is the most linear in the past section. Table I presents the mean Strouhal number $(\overline{S t})$ and the error $(\%)$. Mean Strouhal number $(\overline{S t})$ is the average value of Strouhal numbers for different Reynold numbers at the fix blockage ratio and slit ratio. It can be noticed that the blockage 0.24 is minimum in error percentage. The three blockages: $0.23,0.24$, and 0.26 , that give least error were selected for further studying on the effect of slit to diameter ratio $(s / d)$.

\section{B. Analysis on Slit to Diameter Ratio (s/d Ratio)}

The bluff body used in this case is slit-body shape. It is needed, therefore, to analysis on the effect of slit to Strouhal number. The slit ratios from 0.10 to 0.16 were used for the present work. The $d / D$ ratios that give minimum error by Table I have been analyzed with different slit ratio.

As shown in Fig. 3, the Strouhal number varies not only by the blockage but also by the slit. Although $d / D=0.26$ is least error for $s / d=0.10, d / D=0.23$ gives minimum error at $s / d=0.16$. Overall, $d / D=0.24$ with $s / d=0.14$ has the least in error among the tested ratios. On the other hand, the design with blockage ratio 0.24 and slit ratio 0.14 has the higher linearity than other design ratios. So, we choose this design as optimum by the linearity of Strouhal number against Reynold number.

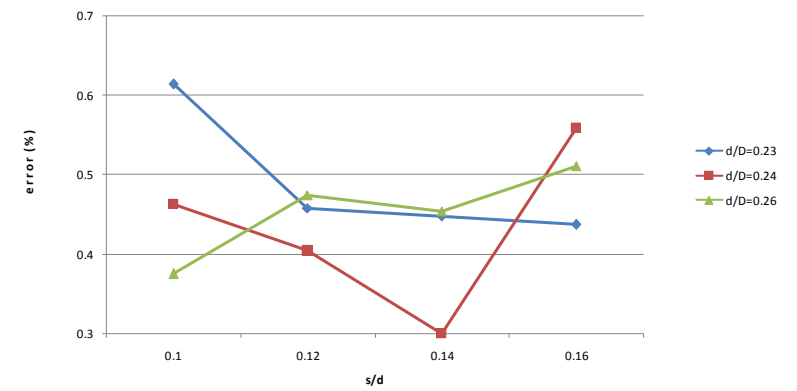

Fig. 3. Variation in error (\%) at different slit to shedder diameter ratio (s/d) for blockages $0.23,0.24, \& 0.26$.

\section{Analysis on Apex Angle $(\theta)$}

It is remained to analyze the effect of apex angle to Strouhal number. Apex angle means the angle facing with incoming flow or outgoing flow. While the other ratios are kept constant, just changing in apex angle seems very sensitive to vortex shedding. The smaller the angle, the more slender the shedder body is. It is well know that longer and more slender shedding bodies result in weaker and less coherent shedding [10]. Therefore, we took only three angle, $45^{\circ}, 60^{\circ}$, and $90^{\circ}$ for this work.

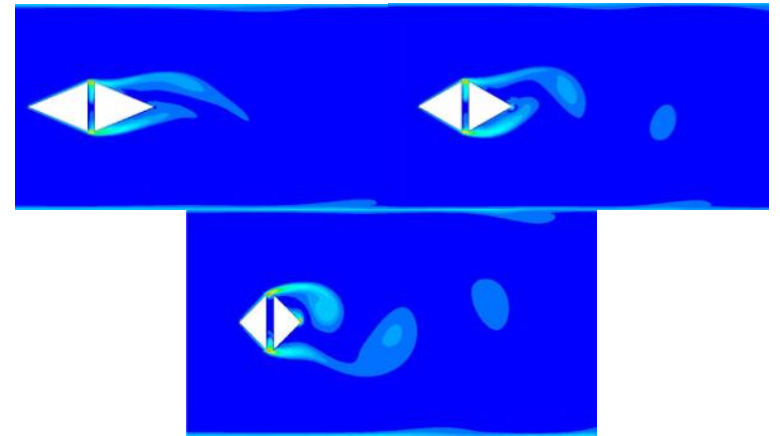

Fig. 4. Vorticity contour for different $\theta$ at $\operatorname{Re}=60000$ (a) $\theta=45^{\circ}$ (b) $\theta=60^{\circ}$ (c) $\theta=90^{\circ}$ 
The simulation results show that $45^{\circ}$ angle bluff body is poor in linearity in Strouhal number with an error of over $5 \%$. For $90^{\circ}$ shedder, it can generate strong vortex shedding with good accuracy of $1.4266 \%$ error. However, its percentage is still high compared with the $60^{\circ}$ shedder $(0.3 \%)$ that also generates the strong vortex shedding. The vorticity contour lines at $\mathrm{Re}=60000$ for all cases are presented in Fig. 4 as a better representation of the Von Kármán vortex street.

By the simulation results above, we chose the interested bluff body with the parameters of 0.24 blockage ratio, 0.14 slit ratio and $60^{\circ}$ apex angle as the best design by the linearity in Strouhal number.

\section{CONCLUSION}

The unsteady flow past a new vortex shedder design has been investigated numerically. The optimization was achieved by the linearity of Strouhal number against Reynold number. The linearity is determined by the error $(\%)$ calculated from equation (10). To obtain the accurate shedding frequency, we take the average values from the 10 shedding cycles. For one shedding cycle, 90 time steps are used so as to minimize the time discretization error.

In the present work, the bluff bodies with blockage ratio (0.16-0.30) were considered and the slit to shedder diameter ratio (s/d) 0.10-0.16 were used. And, we took only three apex angle, $\left(45^{\circ}, 60^{\circ}\right.$ and $\left.90^{\circ}\right)$ to analysis as longer and more slender shedding bodies result in weaker and less coherent shedding.

By the numerical results, it has found that the slit-diamond shape with blockage ratio (d/D) 0.24 , slit to shedder diameter ratio (s/d) 0.14 and apex angle $(\theta) 60^{\circ}$ is the best design by the point of linearity in Strouhal number against Reynold number. Furthermore, this bluff body design can give the following advantages:

- The design is simple. So, it is easy to manufacture.

- As there are no curve surfaces, manufacturing errors can be reduced.

- As it is a sharp-edge vortex shedder, it will be sure for the fixed separation point irrespective of Reynold number range.

- It can be used in the flow rate as low as Reynold number of 2400 with well accuracy.

\section{REFERENCES}

[1] E. Achenbach, "Distribution of local pressure and skin friction around a circular cylinder in cross-flow up to $\mathrm{Re}=5 \mathrm{X} 106$, , Journal of Fluid Mechanics, vol. 34, no. 625-639, 1968.

[2] G. L. Pankanin, "The vortex flowmeter: Various methods of investigating phenomena," Measurement Science and Technology, vol. 16, pp. R1-R16, 2005.

[3] A. Venugopal and S. V. Prabhu, "Review on vortex flowmeter Designer perspective," Sensors and Actuators, vol. 170, pp. 8-23, 2011.

[4] A. Roshiko, "Experiments on the flow past a circular cylinder at very high Reynolds number," Journal of Fluid Mechanics, vol. 10, pp. 345-356, 1961.

[5] M. T. Y. Terao, "Standardization of vortex shedding flowmeter," presented at 4th International Symposium on Fluid Flow Measurement, Denver, Colorado USA, June 27-30, 1999

[6] T. Igarashi, "Flow resistance and strouhal number of a vortex shedder in a circular pipe," JSME International Journal, vol. 42, no. 4, 1999.

[7] B. K. Gandhi, S. N. S. V. Seshadri, and J. Singh, "Effect of bluff body shape on vortex flowmeter performance," Indian Journal of Engineering \& Materials Sciences, vol. 11, pp. 378-384, 2004.

[8] FLUENT 6.3 User's Guide. Fluent Inc., 2006

[9] S. H. Johnsson et al., "Numerical simulation of vortes shedding past triangular cylinders at high Reynold numbers using a k-e turbulence model," International Journal for Numerical Methods in Fluids, vol. 16, pp. 859-878, 1993

[10] R. C. Baker, "Flow measurement handbook: Industrial design, operation principles," Performance, and Applications, Cambridge University Press, 2000.

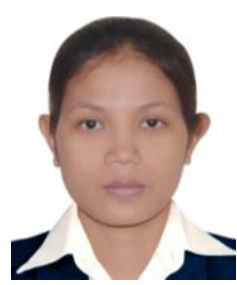

Su Myat Nyein was born in 1984, in Magway region, Republic of the Union of Myanmar. She has been graduated with the bachelor of engineering (mechanical) degree in 2006 and the master of engineeing (mechanical) degree in 2009 from Technological University (Pakoku), Republic of the Union of Myanmar. At present, she is pursuing her Ph.D degree at College of Mechanical and Electrica Engineering, Harbin Engineering University, China. Her current research interests are parametric optimization and fluid flow analysis.

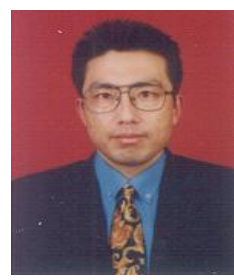

He Xu was born in 1964, Heilongjiang province, China. He received his BS in fluid transmission and pressure control from Zhejiang University, Hangzhou, China in 1987, and the MSc and PhD degrees in mechatronic control and automation from Harbin Institute of Technology, Harbin, China in 1990 and 2006, respectively. He has participated in some mass transit projects at home and abroad. He is now a professor in Harbin Engineering University. His research interests include special micro robots in extreme environment, analysis of multi-physics coupling in water hydraulic system, mechatronic and vision systems. He has published more than 30 refereed papers in technical journals and conference proceedings. 



\section{Industrial Engineering and Optimization}

Management 
